\title{
Comprendre les dynamiques de la gouvernance du secteur de la sécurité en Afrique de l'Ouest
}

\author{
Alan Bryden* et Fairlie Chappuis ${ }^{\dagger}$ \\ ${ }^{*}$ Directeur Adjoint et Chef de la Division de Partenariats Public-Privé au \\ Centre pour la Contrôle Démocratique des Forces Armées (DCAF) \\ ${ }^{\dagger}$ Responsable de Programme dans la Division Recherche au Centre pour la \\ Contrôle Démocratique des Forces Armées (DCAF)
}

\section{Les promesses de la bonne gouvernance pour la sécurité, le développement et la démocratie en Afrique de l'Ouest}

Si lère ouverte par l'après-guerre froide avait suscité un nouvel espoir pour le développement, la sécurité et la démocratie en Afrique de l'Ouest, ces perspectives se sont assombries durant la première décennie de ce millénaire. L’essor de léconomie nigériane a certes déplacé le centre de gravité économique du continent africain vers la région de l'Afrique de l'Ouest. Mais la croissance économique na pas tenu la promesse d'un avenir meilleur pour une génération de jeunes ouest-africains privés d'une éducation solide et confinés à la précarité d’emplois informels. Si les pressions sociales suscitées par ces espoirs déçus n’ont pas, jusquà présent, dégénéré en des conflits violents de l’ampleur de ceux observés dans les années 1990 et au tournant des années 2000, ces tensions ont néanmoins contribué à des crises internes provoquées par lopposition de groupes d'insurgés, de séparatistes et de terroristes (ainsi que des éléments de la criminalité organisée) à lautorité centrale de l'Etat. Dans le même temps, les crises sécuritaires auxquelles les populations sont confrontées au quotidien - du fait de la croissance de la criminalité et des dysfonctionnements des services de

Comment citer ce chapitre du livre:

Bryden, A et Chappuis, F. 2015. Comprendre les dynamiques de la gouvernance du secteur de la sécurité en Afrique de l'Ouest. Dans: Bryden, A et Chappuis, F (dir. publ.) Gouvernance du secteur de la Sécurité : Leçons des expériences ouest-africaines, Pp. 1-21. London: Ubiquity Press. DOI: http://dx.doi.org/10.5334/bav.a. Licence: CC-BY 4.0. 
sécurité fournis par l'Etat - continuent de freiner le potentiel de développement économique et de renforcement de la démocratie.

Si les normes de la gouvernance démocratique semblent sêtre intégrées aux pratiques d'un certain nombre de pays (comme en témoigne l'alternance démocratique pacifique et contrôlée au Libéria en 2011, au Sénégal en 2012, ou au Nigeria en 2015), les bouleversements en Guinée en 2008, ou au Mali en 2012, montrent également que la compétition violente pour le contrôle des pouvoirs de l'Etat reste un risque potentiel. Le caractère interdépendant de ces différents défis apparaît dorénavant plus clairement, ce qui souligne les liens étroits entre démocratie, développement et sécurité. Ainsi, les événements dans le nord du Nigeria et du Mali ou encore au Bénin, au Niger et dans d'autres Etats de la région ont montré que le sous-développement peut être une cause directe d'insécurité et menacer la gouvernance démocratique. Ces exemples soulignent également que ce qui peut apparaître de prime abord comme un problème local ou infra-national peut rapidement s'intensifier pour non seulement atteindre le cœur politique d'un Etat, mais également dépasser les frontières et devenir un risque pour la sécurité régionale. De même, l’instabilité générée par les luttes de pouvoir entre les élites au sommet de l'Etat peut rapidement bloquer les processus démocratiques, en sapant la légitimité de l'Etat et la confiance économique nécessaire au développement: la Guinée, la Guinée-Bissau comme la Côte d'Ivoire ont été confrontées à des crises de cet ordre durant la première décennie des années 2000.

Au niveau mondial, plusieurs initiatives ont été prises pour répondre à ce bilan décevant. Les Objectifs du Millénaire pour le développement ont souligné l'importance de la réduction de la pauvreté pour la prévention des conflits et ce prédicat a été intégré dans des initiatives internationales telles que le New Deal pour l'engagement dans les Etats fragiles, qui a été adopté au cours de la décennie suivante et qui a inclus 19 Etats fragiles ou en conflit, puis tous les pays donateurs de l'OCDE (Partenariat de Busan pour une coopération efficace au service du développement 2011; Nations Unies 2000; Déclaration de Paris sur l'efficacité de l'aide au développement 2005; Programme d'action d'Accra 2008; Dialogue international sur la consolidation de la paix et de renforcement de l'Etat 2011). Le rapport 2011 sur le développement dans le monde de la Banque mondiale s'inscrit dans cet élan politique en proposant une approche plus holistique qui intègre les recherches les plus actuelles en matière de démocratie, de développement et de sécurité au sein d'un programme d'action visant à instaurer «la sécurité des citoyens, la justice et l'emploi» par le biais d'institutions plus fortes et plus légitimes (rapport 2011 sur le développement dans le monde). En 2015, ce programme d'action a franchi une nouvelle étape en identifiant la mise en place d'institutions responsables et inclusives comme un but explicite des objectifs de développement durable et en liant ce dernier à la prévention des conflits et à la promotion de la paix (Objectifs de développement durable 2015). 
La gouvernance émerge comme le concept central reliant démocratie, développement et sécurité. Les travaux de recherche stratégique et académique en matière d'économie du développement, de prévention des conflits et de démocratie convergent ainsi en soulignant que la qualité de la gouvernance a un impact direct sur la trajectoire politique nationale (voir par exemple Halperin et al 2010; Collier 2007). En cherchant à appliquer ces hypothèses aux politiques et pratiques de la gouvernance, ces recherches ont identifié un certain nombre de caractéristiques institutionnelles qualifiées de "bonne gouvernance», qui contribuent à favoriser le développement, la sécurité et la démocratie. Si certains aspects spécifiques de ce programme de bonne gouvernance varient en fonction des contextes et des institutions, les éléments fondamentaux incluent: la redevabilité, l'efficacité, l'efficience, la transparence, l'inclusivité, l'équité et l'Etat de droit (Shabbir Cheema 2005).

Lobjectif de la réforme du secteur de sécurité (RSS) est d'appliquer les principes de bonne gouvernance au secteur de la sécurité. La RSS vise à renforcer la sécurité de l'Etat et de la population en améliorant la redevabilité et l'efficacité en matière de prestation de services de sécurité, et de contrôle et de gestion de ce secteur, dans un cadre reposant sur le contrôle démocratique, le respect des droits humains et l'Etat de droit (Bryden et Hänggi 2004; Hänggi 2003; 2004). Lapplication des principes de bonne gouvernance au secteur de la sécurité souligne notamment que, pour être en mesure de consolider la démocratie, le développement et la sécurité, l'Etat ne doit pas être uniquement préoccupé par les questions de défense nationale et de sécurité de l'Etat, mais il doit aussi répondre aux besoins de sécurité humaine, en s'assurant que la population vive à l'abri de la peur (Krause 2006). Le concept de sécurité humaine inscrit l'objectif d'une prestation de services de sécurité et d'une gestion et d'un contrôle efficaces et responsables de ce secteur par l'Etat dans un cadre de gouvernance démocratique, de respect des droits humains et d'Etat de droit.

La RSS a tendance à se focaliser sur les institutions comme modalité de renforcement du monopole légitime de l'Etat en matière de recours à la force. North (1990) a été l'un des premiers auteurs à souligner le rôle des institutions dans la prestation de services publics et cette hypothèse a été renforcée par Robison et Acemoglu (2012) qui ont mis en avant l'importance de disposer d'institutions accessibles et transparentes, tandis que Fukuyama (2013) a exploré les capacités de l'Etat en matière de prestation de services publics. Si l'ensemble des institutions du secteur public ont un rôle à jouer en matière de démocratie, de développement et de sécurité, les obligations qui incombent aux autorités étatiques spécifiquement chargées de la prestation de services de sécurité et de leur gestion et contrôle présentent des défis particuliers. La bonne gouvernance du secteur de sécurité joue un rôle particulièrement important pour surmonter les défis auxquels sont actuellement confrontés les Etats d'Afrique de l'Ouest et le présent volume s'attache donc aux caractéristiques d'une bonne gouvernance de ce secteur. 


\section{Objectifs de cet ouvrage}

Etant donné le rôle crucial que joue la bonne gouvernance du secteur de la sécurité pour la démocratie, le développement et la sécurité, il est particulièrement important, pour mettre en œuvre des réformes, de comprendre les dynamiques de la bonne ou de la mauvaise gouvernance. Ce livre se focalise sur la nature de la gouvernance du secteur de la sécurité en Afrique de l'Ouest en examinant des moments particuliers et des agents clés du processus de réforme dans six Etats de la région. ${ }^{1}$ Ces différentes études de cas particuliers soulignent ensemble le caractère holistique de la gouvernance du secteur de la sécurité et de la dynamique du processus de réforme dans divers environnements nationaux ayant chacun des caractéristiques propres.

Ce volume diffère des études généralement consacrées à la RSS en ce qu'il ne cherche pas à évaluer au niveau macro et national la réforme dans son ensemble et sur le long terme; il ne vise pas non plus à proposer une analyse opérationnelle des défis sécuritaires actuels. Ce volume s'attache plutôt à retracer les dynamiques essentiellement politiques de la gouvernance du secteur de la sécurité et souligne la nécessité de comprendre ces dernières dans les stratégies, la planification et la mise en œuvre des processus de RSS. Nous n'avons pas, à cette fin, privilégié l'uniformité des analyses mais avons cherché plutôt à recueillir les analyses et points de vue d'individus dont lopinion est particulièrement éclairante du fait de leur connaissance du contexte local. Les auteurs qui ont contribué à ce volume présentent donc les perspectives d'acteurs faisant partie du cercle des «initiés» aux questions de sécurité en se fondant sur leurs connaissances et expériences personnelles. Cette approche a un certain nombre d'implications. Il convient notamment de souligner que ces études de cas ne visent pas à présenter une vision équilibrée qui évalue les avantages et les inconvénients de ces différentes perspectives. Ces études de cas reflètent plutôt les expériences vécues, les convictions personnelles et les parti-pris des auteurs qui ont contribué à ce volume.

La sécurité reste un sujet tabou dans de nombreux contextes nationaux sur le continent africain. Afin de mobiliser des parties prenantes potentielles et de bâtir un large soutien en faveur de la RSS, il est nécessaire de démystifier le secteur de la sécurité. Cela requiert une compréhension approfondie du contexte. C'est la raison pour laquelle les auteurs qui ont contribué à ce volume ont accordé une grande importance à l'histoire politique qui soustend, dans chaque pays examiné, les dynamiques actuelles de la gouvernance du secteur de la sécurité. Un message clé ressort de ces études de cas: pour comprendre les opportunités de réforme et les contraintes qui pèsent sur elle, il est essentiel que les parties prenantes nationales (et les partenaires externes qui veulent soutenir un processus de RSS axé sur la gouvernance) prennent en compte les dynamiques historiques profondes qui ont façonné le secteur de la sécurité au niveau national. Les études de cas réunies dans ce volume visent 
à identifier des leçons pratiques pour favoriser une telle compréhension et promouvoir des changements positifs. Lorsqu’elles sont examinées en parallèle, ces études de cas proposent donc une analyse des tendances émergentes plus générales, en mettant en lumière des orientations futures pour la RSS qui sont présentées dans la conclusion de ce volume.

En se focalisant sur les micro-dynamiques de la réforme, ce volume pose comme postulat la nécessité d’appréhender différemment les critères de succès et déchec d'une réforme du secteur de sécurité. Cette approche est innovante, en particulier en ce qu'elle souligne que l'importance de certains moments spécifiques et agents de changement influents ne peut être pleinement perçue qu'a posteriori. Ainsi, dans un contexte de crise politique voire de conflit violent, les changements potentiellement transformationnels apparaissent souvent comme des éléments isolés, superficiels ou insignifiants. Leur caractère potentiellement transformationnel est donc éludé. Envisager le changement à partir de cette perspective innovante permet d'identifier le succès et l'échec à des moments et dans des lieux inattendus.

Cette approche permet également de corriger la tendance dans la littérature à idéaliser les conditions du succès, tout en négligeant les leçons tirées des échecs. Notre approche tient compte du fait que, bien que les exemples de changements transformationnels positifs soient importants, des leçons utiles peuvent aussi être tirées d'exemples de revirements, de stagnations ou de réformes inabouties. Certaines des études de cas rassemblées dans ce volume soulignent ainsi que les parties prenantes locales ont pu accorder, à des moments particuliers, une importance moindre à la réforme. Les chapitres ont été distribués de façon à tirer les leçons des expériences de pays ayant connu des évolutions politiques diverses: le Ghana, la Guinée, le Libéria, le Mali, le Nigeria et le Sénégal. Cette sélection couvre un éventail de contextes: la transition et la consolidation démocratique, l'après-conflit et le recul du processus démocratique. Cet éventail détudes de cas offre une base de comparaison instructive en ce qu'elles soulignent les enseignements issus d'expériences de progrès ainsi que de reculs.

En résumé, les études de cas qui constituent l'essentiel de ce volume soulignent l'utilité d'adopter une perspective méthodologique qui met l'accent non pas sur le plan national mais plutôt sur les micro-dynamiques de la réforme institutionnelle dans le contexte politique immédiat. A travers les yeux et les expériences d'acteurs locaux, ces études de cas analysent les succès de modeste ampleur de la RSS ainsi que les occasions manquées de faire en sorte que la réforme ait des effets transformationnels plus larges. En se basant sur l'analyse de moments potentiellement transformationnels de processus de réforme par des experts nationaux éminents ayant une expérience personnelle de ces dynamiques, ce volume montre comment les initiatives de RSS influencent les dynamiques de gouvernance du secteur de la sécurité d'une manière significative, même si elle est limitée, et il tire des leçons concrètes et pratiques de ces expériences de réforme au niveau national. 


\section{Face à un bilan décevant : comprendre les difficultés de parvenir à des changements transformationnels en Afrique de l'Ouest}

Malgré les efforts importants et certaines avancées, la plupart des Etats africains sont loin d'avoir atteint l'objectif d'assurer une gouvernance plus démocratique du secteur de la sécurité. Les caractéristiques de la gouvernance du secteur de la sécurité sont déterminées par les dynamiques historiques, politiques et économiques complexes propres à la structure de chaque situation nationale - et infranationale. Pour autant, les études de cas rassemblées dans ce volume permettent d'identifier des expériences communes et des tendances régionales.

L'héritage de l'Etat colonial et post-colonial influe de manière déterminante sur la gouvernance du secteur de la sécurité. Si les pays d'Afrique de l'Ouest ont connu diverses expériences coloniales, nombre d’entre eux ont l'expérience commune d'une autorité politique centrale illégitime et aux visées spoliatrices. Cet héritage a prédisposé les institutions du secteur de la sécurité en Afrique de l'Ouest à se focaliser sur l'extraction de ressources et le contrôle de la population, et ces caractéristiques se sont perpétuées dans le contexte moderne. D’ailleurs, au cours de leur histoire, la population et les dirigeants de ces Etats peuvent n'avoir eu pour seul modèle de prestation de services de sécurité que celui d'une autorité étatique autoritaire et aux visées spoliatrices. Dans un tel contexte social, l'amélioration des services de sécurité fournis par l'Etat ne peut se réduire à un simple problème de réforme technique ou d'amélioration des équipements ou des formations: il faut repenser la raison dêtre des prestataires de sécurité étatiques depuis la base jusquau sommet en se fondant sur une vision nouvelle et différente de ce quest la sécurité et au nom de qui elle est rendue.

Si l'héritage du colonialisme a été perpétué par les institutions de sécurité modernes en Afrique de l'Ouest, ces tendances ont aussi été exacerbées par le processus inabouti de démocratisation dans la région. Les régimes politiques post-indépendance ont adopté des pratiques de gouvernance non libérales tout en bénéficiant d'un important soutien extérieur. Ils ont également favorisé la mise en place de secteurs de la sécurité prédateurs, ne répondant pas de leurs actes et axés sur la sécurité du régime/de l'Etat. Dans de nombreux cas, ce déficit démocratique a contribué à la perpétuation de ces régimes pendant des décennies, ce qui a profondément enraciné ces pratiques de prédation. Le pouvoir législatif s'est retrouvé assujetti à un exécutif puissant et les membres du pouvoir judiciaire sont devenus les serviteurs du pouvoir d'Etat au lieu dêtre les gardiens de l'Etat de droit. Dans ces conditions, peu d'Etats ont développé des systèmes efficaces de contrôle démocratique civil et la sécurité nationale est devenue une sphère d'influence exclusivement réservée aux acteurs politiques et aux hommes en uniforme les plus puissants. 
Face à des secteurs de la sécurité étatique servant les intérêts du pouvoir, les populations ont pris des mesures pour assurer leur propre sécurité. Les individus dotés de moyens financiers ont fait appel à des prestataires de sécurité privés, alors que les citoyens démunis ont dû assurer eux-mêmes leur protection. De ce fait, alors que les Etats de l'Afrique de l'Ouest disposaient d'un secteur de la sécurité de taille importante et parfois doté de ressources conséquentes, les besoins sécuritaires quotidiens d'une majorité de la population ouest-africaine étaient assurés par des prestataires de sécurité communautaires non étatiques ou par des entreprises de sécurité privées.

Ce contexte de difficultés économiques, d’inégalités sociales, et de privation des droits politiques a fait le lit de conflits armés tout en générant des tensions sociales qui ont favorisé la criminalité et la violence politique. Les Etats affaiblis par des systèmes politiques clientélistes inefficaces ne disposaient pas des capacités institutionnelles nécessaires pour répondre efficacement aux défis auxquels ils étaient confrontés, et leurs capacités institutionnelles et humaines se sont encore davantage amoindries du fait de ces problèmes. La légitimité de l'autorité de l'Etat étant rongée par l'inefficacité et par des pratiques de prédation, le secteur de la sécurité en est venu, lui aussi, à symboliser l'illégitimité de ce pouvoir et la dureté de la répression étatique.

\section{Lémergence de l'idée de RSS}

La RSS a émergé à la fin des années 1990 comme réponse aux dysfonctionnements de la gouvernance du secteur de la sécurité et à leurs conséquences. Promue tout d'abord par les agences de développement européennes, la RSS est rapidement devenue un pilier des stratégies multilatérales en matière de prévention des crises, de consolidation de la paix et de développement qui ont été promues par des organisations telles que les Nations Unies, l'Union africaine, la CEDEAO, l'Union européenne, la Banque mondiale et l'OCDE (Conseil de sécurité de l'ONU 2014; Commission de l'Union africaine 2013; Ball, 2001; Aning, 2004; Conseil de l'Union européenne 2005; Conseil de l'Union européenne, 2006). Si la RSS est souvent perçue comme une prescription imposée de l'extérieur aux pays bénéficiaires, en particulier dans les situations de postconflit, cette conception de la RSS va en fait à l'encontre des objectifs, des principes et des expériences passées en matière de RSS. Cela s'explique par des raisons à la fois d'ordre pragmatique et normatif. Du point de vue pragmatique, les stratégies de réforme imposées de l'extérieur ont été à maintes reprises vouées à l'échec, parce qu'elles nétaient pas adaptées au contexte local ou nétaient pas ancrées dans le contexte local de la gouvernance. Sur le plan normatif, les principes de bonne gouvernance sont incompatibles avec l'imposition d'une stratégie de réforme. En outre, l'histoire a montré que les seuls changements durables en matière de gouvernance du secteur de la sécurité sont intervenus 
lorsque le programme de réforme était placé sous la houlette d'un leadership national fort: des exemples aussi divers que ceux de lère Reformasi dans l'Indonésie post-Suharto et de la transition démocratique de l'Afrique du Sud post-apartheid démontrent l'efficacité d'une forte volonté politique en faveur de la réforme (Cawthra et Luckham 2003). ${ }^{2}$ Ces expériences passées soulignent qu'il est essentiel que les parties prenantes nationales et locales fassent preuve de leadership et s'investissent dans ces processus de réforme, pour garantir une amélioration durable et réelle de la gouvernance du secteur de sécurité - même si, dans la pratique, les stratégies de réforme laissent souvent à désirer (Nathan 2007; Donais 2008; 2009).

En s'appuyant sur une conception de la sécurité fondée sur la notion plus large de gouvernance, la RSS rassemble tous les acteurs concernés par la sécurité, qu'il sagisse des prestataires de services de sécurité, des agents chargés de leur contrôle ou de leurs bénéficiaires et ce, indépendamment du fait que ces acteurs soient étatiques ou non-étatiques (Chappuis et Hänggi 2013). Cette conception de la RSS axée sur la gouvernance implique une approche holistique de la réforme et peut donc recouvrir un large éventail de mesures, y compris: lélaboration de cadres législatifs plus robustes régissant la prestation de services de sécurité et la gestion et le contrôle de ce secteur; l'adoption de réformes axées sur des institutions de sécurité spécifiques telles que la police, les autorités militaires, les services de renseignement ou les agents chargés du contrôle aux frontières; et la mise en place d’organes et de mandats aux fins spécifiques de contrôler la prestation des services de sécurité, telles que des commissions des droits de l'homme ou des institutions de médiation, des organes parlementaires ou le secteur de la justice (OCDE-CAD, 2007; Groupe de travail sur le RSS de l'ONU, 2012; DCAF, 2015; Nations Unies, 2008; 2013). En outre, le processus de RSS tient également compte du fait que sécurité et justice sont intrinsèquement liées et il englobe, par conséquent, le secteur de la justice. Cette conception holistique constitue le socle conceptuel de la RSS en tant que processus global traitant de tous les aspects du recours à la force (qui, comment et au nom de quelle autorité la force est utilisée). C'est cette caractéristique qui distingue la RSS des autres formes d'assistance ou de développement des capacités en matière de sécurité - la RSS vise, en tous les cas, à améliorer la redevabilité et l'efficacité des institutions qui assurent ces services. Une réforme qui privilégierait un aspect au détriment d'un autre serait donc incompatible avec le concept de RSS (Chappuis et Hänggi 2009).

Des processus de RSS ont été lancés dans différents Etats de la région, dans les années 2000, pour répondre aux défis fondamentaux de gouvernance auxquels étaient habituellement exposés de nombreux pays de l'Afrique de l'Ouest. En Sierra Leone, au Libéria, en Guinée-Bissau et en Côte d'Ivoire, diverses tentatives de RSS ont été engagées, avec un soutien international important, dans le cadre de processus de construction de la paix suite à des conflits civils (voir respectivement, Bryden et al 2008; Albrecht et Jackson 2009). Au Nigeria, au Bénin, au Mali et au Ghana, des processus de RSS ont été initiés dans le contexte 
de transitions démocratiques (voir également Bryden et N’Diaye 2011). Si la RSS n'est pas spécifique à l'Afrique, de nombreuses expériences importantes dans ce domaine ont eu lieu sur ce continent et les Etats de l'Afrique de l'Ouest ainsi que l'organe régional de la CEDEAO ont joué un rôle clé dans lélaboration de ce concept et le développement de la pratique en matière de RSS. La bonne gouvernance du secteur de la sécurité sous-tend plusieurs instruments de cet organe régional, notamment le Protocole relatif au mécanisme de prévention, de gestion, de règlement des conflits, de maintien de la paix et de la sécurité (CEDEAO 1999), le Protocole additionnel sur la démocratie et la bonne gouvernance (CEDEAO 2001), l'Acte additionnel portant code de conduite des forces armées et services de sécurité (CEDEAO 2011) et plus récemment le Cadre de politique régional sur la réforme du Secteur de la sécurité et de la gouvernance (CEDEAO 2014; voir également, Uzoechina 2014).

Prises conjointement, ces diverses expériences de réformes à léchelle régionale ont apporté un certain nombre déclairages sur les caractéristiques habituelles de la gouvernance du secteur de la sécurité dans de nombreux contextes de réforme. Les analyses fournies en conclusion de ce volume développent en détail ces caractéristiques mais, à ce stade, il convient de noter que la volonté politique au niveau de l'exécutif joue un rôle déterminant (ou, à tout le moins, disproportionné) sur lévolution de la réforme. Linfluence prédominante de l'exécutif sur le processus de réforme tient au fait que les questions de sécurité en général sont souvent considérées comme un domaine réservé sur lequel peu d'hommes politiques civils ont une quelconque influence. Il est donc absolument essentiel que le programme de réforme soit soutenu par les élites du secteur de la sécurité pour que la RSS puisse avancer. En retour, le contrôle étroit exercé par l'exécutif et les forces de sécurité sur le pouvoir et sur les affaires sécuritaires permet d'expliquer la faiblesse notable et persistante du pouvoir législatif dans la région. Que ce soit du fait d'un manque d'autorité politique ou en raison de ressources humaines et financières insuffisantes pour remplir le mandat démocratique confié aux parlements, tous les pays examinés dans ce volume sont caractérisés par une faiblesse du contrôle législatif en matière de sécurité. Ce déficit de contrôle démocratique officiel peut dans une certaine mesure être compensé par une participation forte de la société civile et, à cet égard, de nombreux contextes en Afrique de l'Ouest sont caractérisés par un plaidoyer actif de la société civile en faveur d'une meilleure gouvernance du secteur de la sécurité.

\section{La réforme du secteur de la sécurité: des dimensions négligées}

Etant donné le déficit de gouvernance qui caractérise de nombreux systèmes politiques de la région, il n’est pas étonnant que la RSS n’ait pas généré des effets transformationnels. Comme le notent Hutchful et Luckham (2010), la perspective d'une bonne gouvernance du secteur de la sécurité est très éloignée de 
la réalité dans la plupart des contextes africains. C'est la raison pour laquelle, pour aboutir à une RSS réellement efficace, il est nécessaire de procéder, dans de nombreux Etats, à une transformation radicale des structures du pouvoir et de la gouvernance. Cette nécessité de transformer radicalement les structures politiques, historiques et économiques les plus fondamentales de la gouvernance afin d'atteindre les objectifs d'une bonne RSS a été considérée comme une «dimension spécifiquement africaine» de la réforme du secteur de la sécurité, et celle-ci a même été qualifiée de «transformation du secteur de la sécurité» (Bryden et Olonisakin 2010).

Il y a cependant une divergence évidente entre ces aspirations de la RSS et la forme que ce type de processus prend invariablement: Bryden et Olonisakin soulignent explicitement que «la transformation du secteur de la sécurité, malgré sa vocation radicale, a tendance à avoir un caractère progressif et processuel» (Bryden et Olonisakin 2010: 22). Or, les approches actuelles en matière de RSS reposent sur le prédicat selon lequel la RSS peut rapidement modifier en profondeur les conditions de la gouvernance et que les résultats de ces stratégies de réforme, en cas de succès, doivent être visibles. Cette approche ne tient pas compte du fait que le changement est un processus, et cela entraîne des attentes irréalistes et conduit à se focaliser sur les aspects les moins susceptibles d'avoir un impact. De manière symptomatique, de nombreuses stratégies de RSS mettent ainsi l'accent sur la formation et léquipement des forces de sécurité, tout en négligeant les questions de gouvernance démocratique. Or, dans ce domaine, une approche plus nuancée s'impose pour comprendre la nature de la RSS et lévaluer en tant que processus itératif et graduel.

La prise en compte du caractère fragile et progressif de la RSS permet de comprendre et d'évaluer autrement la contribution relative de la RSS à la gouvernance démocratique, à la paix et au développement. Pour appréhender pleinement la nature itérative et transformationnelle de la RSS, il faut opérer un changement de perspective afin de mieux identifier et évaluer l'importance potentielle à long terme des changements progressifs dans le cadre d'un processus continu. Il faut, pour cela, tenir compte du fait que les moments potentiellement transformationnels de la réforme sont marqués par des changements dans la qualité de la gouvernance du secteur de la sécurité qui sont difficiles à identifier comme tels au moment où ils émergent et qui peuvent être facilement inversés si le processus prend des directions imprévues.

Les signes indiquant une transformation significative des déterminants structurels de la gouvernance sont peu visibles et les approches actuelles de la RSS ne permettent pas de les apprécier pleinement. Comme l'importance relative de ces changements modestes qui peuvent conduire à des mutations sur le long terme n'est pas perçue, cela empêche l'adoption d'une stratégie souple et adaptée au déroulement du processus de RSS. L'argument selon lequel les objectifs de la RSS sont définis à l'aune d'un modèle paradigmatique de bonne gouvernance du secteur de la sécurité qu’aucune société ne reflète pleinement 
contient un élément de vérité. Il faut dès lors reconnaitre qu’à la lumière de cette notion idéalisée, tous les efforts de réforme ne peuvent quêtre jugés insuffisants et toutes les mesures de changement laissent à désirer. Du fait de cette tendance à attendre trop et trop vite, les «succès » en matière de RSS sont définis en fonction de critères tangibles ou visibles qui s’avèrent souvent dans la pratique éphémères et transitoires si tant est qu'ils se matérialisent. Les conceptions actuelles en matière de RSS sont donc focalisées sur l'identification des changements de grande ampleur et des pratiques directement visibles plutôt que sur les modifications modestes des normes, des attitudes et des attentes qui peuvent pourtant être le signe d’avancées dans la bonne direction.

Non seulement les signes réels de changements sont de plus petite ampleur que présumé, mais ils sont également susceptibles dêtre littéralement "invisibles ». Les approches actuelles de la RSS ont tendance à mettre l'accent sur les changements visibles dans la prestation de services de sécurité, que ceux-ci se manifestent par des améliorations des infrastructures physiques et des équipements ou par la mise en place d'institutions ou de systèmes nouveaux. Cette optique met l'accent sur les caractéristiques organisationnelles formelles de la gouvernance alors qu'il s'agit souvent d'une façade dissimulant la base normative informelle de la gouvernance du secteur de sécurité: celle qui définit les «règles du jeu» réelles (North, 1990). Cette dimension a été en partie prise en compte dans les approches de la RSS et quelques initiatives innovantes ont tenté d’améliorer la méthodologie de planification, de conception et dévaluation dans ce domaine. Ces initiatives ont, en particulier, intégré la prestation de services de sécurité et de justice non-étatiques dans les cartographies des acteurs et les stratégies de réforme; elles ont également inclus, dans les évaluations de la RSS, des outils de mesure de la satisfaction et de la perception, en s'appuyant sur des méthodes qualitatives telles que les groupes de discussion, les entretiens et les enquêtes de perception (voir par exemple, le CENAP/CREDESS-BDI 2012; Schnabel 2009). Si ces approches innovantes ouvrent des voies intéressantes en matière dévaluation des changements dans la prestation de services de sécurité par l'Etat, les stratégies de réforme restent cependant trop souvent focalisées sur les impacts institutionnels de programmes de réforme par le haut.

Les approches actuelles de la RSS négligent également l'impact des chocs exogènes et endogènes. Ainsi, outre les problèmes liés aux aspects conceptuels de la réforme, il apparaît également clairement que la RSS peut aussi essuyer des revers et de complètes inversions de tendance pour des raisons externes au processus. Pourtant, jusqu’à présent, les approches de la RSS n'ont pas intégré de manière cohérente le fait que la RSS peut être fréquemment lobjet de revirements dans les contextes de transition (Chappuis et Siegle 2015). Le fait que ce facteur ne soit pas pris en compte parait d'autant plus frappant, compte tenu de la fréquence avec laquelle les processus de RSS sont l'objet de revers en raison de causes endogènes à l'environnement politique national ou à la suite de chocs externes. D'un point de vue pragmatique, pour pouvoir identifier 
les opportunités de réforme, il faut préalablement être en mesure de replacer ces reculs et ces revirements dans leur contexte. Du fait de l'absence de prise en compte de ces facteurs extérieurs, la gravité relative des revirements et des échecs est surestimée alors que certains travaux ont bien montré que ce phénomène était récurrent dans les contextes de transitions démocratiques et de conflits (voir Halperin et al 2010; Haggard et Kaufman 1995; Przeworski et al 2000; Collier 2010; Freunda et Melise Jaud 2013). Une approche tenant compte du caractère progressif de la réforme permet au contraire d'évaluer les avancées et les reculs par rapport au contexte de la gouvernance de la sécurité locale et non à l'aune d'un idéal-type de l'Etat.

Pour comprendre les potentialités transformationnelles de la réforme, il est également essentiel de mettre laccent sur les conditions locales de la gouvernance de la sécurité. Contrairement aux dimensions précédemment mentionnées qui n’ont pas été suffisamment prises en compte, les programmes d'action de la RSS ont compris la nécessité de se focaliser sur la gouvernance de la sécurité au niveau local. Les stratégies de réforme doivent en effet tenir compte des déterminants du contexte local tel que constitué par les acteurs, la politique et le contexte social et historique au niveau local; cela souligne à nouveau la nécessité de l'appropriation locale du programme de RSS. Or, une approche tenant compte du caractère itératif de la RSS requiert une analyse bien plus fine du contexte local que ce que les spécialistes de la RSS ont été enclins à faire jusquà présent, étant donné le caractère par définition limité des perspectives externes sur les dynamiques de gouvernance locales (Schroeder et al. 2013). Par conséquent, létude des processus de la RSS a été fortement biaisée au profit d'analyses externes des contextes de réforme qui évaluent des changements superficiels dans l'organisation ou la structure de sécurité à l'aune d'un modèle occidental idéalisé. Pour dépasser les limites de cette perspective biaisée, il convient de procéder à une analyse des processus de RSS fondée sur une compréhension en profondeur du contexte historique, social, économique et politique. Les points de vue et les perspectives des acteurs locaux peuvent contribuer à corriger la tendance générée involontairement par les approches de la RSS pilotées de l'extérieur à émettre des attentes irréalistes.

\section{Un processus itératif et graduel : reformuler l'analyse de la RSS en Afrique de l'Ouest}

Cette critique des approches actuelles de la RSS appelle logiquement à lélaboration d'une perspective analytique de la RSS en Afrique de l'Ouest qui permette d'appréhender le potentiel de ces réformes en termes de changements itératifs et progressifs. Pour pouvoir évaluer adéquatement l'importance relative de ces changements, il est nécessaire d'identifier les rapports de force entre les divers acteurs au sein et au-delà du secteur de la sécurité. Cette analyse doit 
également s'attacher au contexte structurel dans lequel intervient la RSS et qui détermine la gouvernance du secteur de la sécurité. Ceci ne constitue pas en soi une approche nouvelle - les orientations en matière de RSS ont toujours souligné la pertinence de l'analyse contextuelle et de létablissement d'une cartographie des acteurs (voir, par exemple, l'OCDE-CAD 2007). Les auteurs qui ont contribué à ce volume présentent les informations nécessaires pour élaborer une telle analyse en se focalisant sur des moments spécifiques de changement qui peuvent fournir un éclairage important sur les dynamiques en jeu dans le contexte immédiat et plus généralement sur le processus de réforme.

En ce qui concerne tout dabord les déterminants structurels de la gouvernance du secteur de la sécurité, le potentiel de changement doit être évalué en fonction du contexte des interactions complexes entre les dynamiques politiques, sociales, économiques et historiques en jeu. Ce sont ces variables qui déterminent ensemble ce que Schnabel et Born (2011) définissent comme des environnements de réforme permissifs ou non permissifs. Du fait de l'imbrication de ces différentes dynamiques, les contextes de gouvernance sont d'une complexité extrême qui ne saurait être réduite à des généralisations. Etant donnée, précisément, l'extrême complexité et par conséquent le caractère unique de chaque contexte de réforme, pour comprendre les dynamiques de la gouvernance du secteur de sécurité, il faut analyser en profondeur ces processus. La complexité et l'imbrication de ces dynamiques de gouvernance justifient également d'adopter une échelle micro pour analyser les processus de réforme, car ce sont les interactions inter-institutionnelles et interpersonnelles qui apportent l'éclairage le plus pertinent sur cette complexité. Cette étude détaillée peut en retour servir de base pour une analyse comparative, à la fois pour évaluer soigneusement les implications de ces interactions locales sur le processus général de réforme et pour évaluer le caractère extrapolable, ou non, de ces conclusions à d'autres contextes.

Lorsque les conditions politiques, économiques, sociales ou historiques existantes restent stables, la réforme est peu susceptible de déboucher sur une modification du statu quo. Par contre, lorsque ces conditions structurelles sont plus fluides, cela ouvre de nouveaux potentiels. Les phases de transition à la suite d'un conflit offrent l'exemple typique d'un assouplissement des conditions structurelles qui facilite le potentiel de réforme. Durant ces phases, il y a une certaine fluidité des rapports de force, dans la mesure où l'influence des sources traditionnelles de pouvoir est affaiblie, alors que de nouveaux acteurs nont pas encore eu le temps de s'imposer. Cependant, le statu quo dans une situation apparemment stable peut également être remis en cause de façon inattendue lorsqu'un événement particulier - même en apparence insignifiant - provoque un changement susceptible de modifier les conditions structurelles. Dans les études de cas rassemblées ici, de telles dynamiques sont clairement mises à jour lorsque, par exemple, un événement déclencheur rend soudainement possible, voire nécessaire, de transformer radicalement les 
pratiques antérieures. Le nouveau statu quo, produit par ces épisodes cathartiques, peut favoriser la réforme ou au contraire la faire régresser; dans plusieurs des cas présentés ici, ces événements déclencheurs ont été des moments déterminants de la réforme et ont déclenché des processus de changements potentiellement transformationnels. Afin d'appréhender les dynamiques complexes des contraintes structurelles qui déterminent la gouvernance du secteur de la sécurité dans chacune des études de cas rassemblées dans ce volume, les auteurs ont présenté en détail leurs points de vue sur les facteurs sociaux, politiques, économiques et historiques les plus importants qui influent sur la prestation des services de sécurité par l'Etat.

Ces facteurs structurels de la gouvernance du secteur de la sécurité façonnent l'espace sur lequel se joue la compétition politique autour de la réforme et les moments de confrontation entre ces agendas concurrents sont des événements déterminants du processus de réforme. Ces événements peuvent être de petite ampleur, voire d’apparence banale, mais ils affectent néanmoins les modalités dexercice du pouvoir. Une décision, une nouvelle pratique, un changement de politique, un changement d'attitude: a posteriori, ces moments peuvent enclencher des mutations décisives, qui, sur le long terme, entraînent des effets transformationnels. A ce niveau de micro-dynamiques dans le processus de réforme, le changement peut être rapide, une fois les conditions adéquates réunies. De fait, la vitesse à laquelle les réformes peuvent alors être menées n’a dégal que la rapidité avec laquelle la situation peut se détériorer dans les moments de remise en cause du statu quo. Ces moments de changements rapides dessinent un nouveau statu quo, qui va renforcer et consolider les avancées vers une meilleure gouvernance du secteur de la sécurité - ou au contraire les saper et les entraver. La succession continue de ces moments définit la trajectoire globale du processus de réforme.

Lors de ces moments décisifs, certains acteurs spécifiques, en particulier au sein des puissantes élites politiques et du secteur de la sécurité, peuvent avoir une influence disproportionnée en imposant un changement en faveur ou au détriment de la réforme. Si le statu quo demeure, certains acteurs spécifiques peuvent apparemment avoir peu de marge de manœuvre pour influer sur les déterminants de la gouvernance du secteur de la sécurité. Pourtant, ces conditions peuvent changer et parfois assez rapidement; c'est le cas, en particulier, lorsqu'un certain nombre d'acteurs s'unissent au sein d'une coalition suffisamment influente pour bouleverser les pratiques habituelles qui maintiennent le statu quo en place. L'importance de la mise en place de coalitions «suffisamment inclusives » en faveur de la réforme a bien été démontrée et analysée (rapport 2011 sur le développement mondial: 120). Dans les études de cas rassemblées ici, cette dynamique est illustrée par le degré d'influence étonnamment important que la société civile a pu exercer sur des régimes pourtant insensibles et inflexibles aux nécessités de réforme. De même, dans plusieurs de ces études de cas, l'attitude positive ou négative des dirigeants de haut niveau au sein du 
gouvernement a constitué le facteur le plus décisif de progrès ou de blocage de la réforme. C'est parfois simplement la force des paroles qui était mobilisée par ces acteurs pour peser sur le processus, ce qui illustre le poids sous-estimé du dialogue dans la reconfiguration de la gouvernance du secteur de la sécurité. Pour appréhender cette dimension, les auteurs qui ont contribué à ce volume se sont particulièrement attachés à identifier les champions et les détracteurs de la réforme et la manière dont leurs actions façonnaient les moments décisifs du processus de réforme.

Si laction de certains individus peut avoir une influence déterminante sur la trajectoire de la réforme, leur influence est conditionnée par le contexte dans lequel ces individus opèrent et qui détermine leur marge de manœuvre. $\mathrm{Au}$-delà du rôle de ces agents individuels, la nécessité de répondre à une situation d'urgence peut aussi être un moteur important de changement ou, au contraire, maintenir le statu quo. Malgré la tendance dominante à concevoir la réforme comme résultant des actions rationnelles d'acteurs spécifiques (trop souvent externes), il arrive que le processus ne se déroule pas comme prévu, comme c'est souvent le cas dans les environnements complexes. En l'occurrence, le processus de réforme, son contexte et ses résultats peuvent être déterminés par des conséquences inattendues ou des interactions contingentes. Dès lors, pour mieux comprendre la nature de la RSS, il faut analyser également les effets contingents, accidentels ou inattendus qui se produisent souvent à l'échelle micro. Dans ce volume, les auteurs ont retracé la logique des actions de réforme mais ils ont également souligné les nombreux effets non intentionnels, accidentels et contingents qui ont "découplé» le processus de ces effets anticipés. Cette approche offre un éclairage nuancé des raisons pour lesquelles certaines réformes subissent des blocages, malgré tous les efforts déployés par des champions de la réforme, ou à l'inverse connaissent des avancées, en dépit de l'influence des individus réfractaires à ce processus.

Ces différents éléments soulignent à lévidence que, pour rendre compte de manière exhaustive d'un processus de RSS, il faut avoir une connaissance approfondie du contexte politique, social, économique et historique dans lequel il intervient. Non seulement, le moindre détail sur les acteurs ou le contexte peut se révéler déterminant pour expliquer les avancées ou les reculs de la réforme, mais l'identification de ces paramètres essentiels peut varier sensiblement en fonction de la connaissance du contexte. Les études de contextes nationaux reposant sur une expertise et des perspectives locales permettent d'appréhender le contexte de manière plus fine que ne le peuvent les spécialistes externes, car elles apportent potentiellement un éclairage sur des points qui échappent à leur analyse. Cette approche est prometteuse dans la mesure où relativement peu de travaux sur la RSS en Afrique ont été menés par des experts de la région ou des pays concernés (voir par exemple Uvin 2009; Malan 2008; Greene et Rynn 2008; Albrecht et Jackson 2009). Du fait de la prédominance d’analyses externes, la littérature sur la région a tendance à privilégier les perspectives 
externes en mettant fortement l'accent sur les aspects techniques sans consacrer une attention suffisante au contexte politique, social et historique (pour des exceptions notables à cette tendance, voir Bryden et Olonisakin 2010; Bryden et N'Diaye 2011; Bryden et al 2008).

$\mathrm{Au}$ contraire, les études de cas rassemblées dans ce volume ont été préparées par un groupe diversifié d'individus provenant des milieux de la recherche et des cercles politiques et de la sécurité de chacun des pays examinés. En décrivant chaque contexte et en se fondant sur leur point de vue personnel, ces auteurs nous font bénéficier de leur expérience directe en tant que personnes «initiées» aux questions de la sécurité ainsi que de leur connaissance approfondie de l'histoire, de la société et de la politique des pays examinés. Ces experts ont une connaissance de première main du processus de réforme dont ils rendent compte. Leurs études de cas offrent un aperçu personnel de la situation selon la tradition de la «description dense " plutôt que des analyses de cas formelles selon les canons classiques des sciences sociales. Leur statut d'experts nationaux, qui sont eux-mêmes ancrés dans le contexte social et politique particulier dans lequel se déroule la réforme, leur offre un accès privilégié à des dynamiques qui restent invisibles pour des observateurs externes, quel que soit leur degré de connaissance de l'environnement local. Pour autant, cette analyse ne peut pas être séparée du point de vue, de l'expérience et des prédispositions des auteurs. De ce fait, ces chapitres constituent des études de cas de la dynamique de réforme fondées sur une perspective authentiquement nationale et personnelle.

Les auteurs qui ont contribué à ce volume se sont focalisés sur les dynamiques institutionnelles de la gouvernance de la sécurité et sur la nature de la prestation des services de sécurité par l'Etat. En ce qui concerne tout d'abord la dynamique institutionnelle de la gouvernance de la sécurité, il apparaît clairement que la nature transformationnelle de la RSS implique une transformation de l'équilibre des pouvoirs entre les diverses parties composant le gouvernement; de la relation entre le secteur de la sécurité et le pouvoir civil; de l'équilibre des pouvoirs entre les organes de contrôle civils; et des dynamiques de contrôle et de réactivité entre l'Etat et la population. Les programmes de RSS ont souvent été critiqués parce qu'ils privilégient les réformes dites techniques et à plus court terme au détriment des dimensions touchant à la gouvernance. C'est la raison pour laquelle plusieurs des études de cas rassemblées ici se focalisent explicitement sur les aspects institutionnels de la gouvernance - même si les initiatives de RSS dans les pays concernés sont plus souvent associés à des programmes ambitieux visant à «former et équiper» les forces de sécurité. Ainsi, par exemple, T. Debey Sayndee offre un nouvel éclairage du processus de RSS au Libéria en examinant ce processus au travers des développements législatifs, alors que ce cas est habituellement traité comme un exemple de réforme de la police et de l'armée. De même, Emmanuel Kwesi Aning met l'accent sur l'exemple peu connu de la réémergence du Conseil de police au Ghana, alors que la tendance était de se 
focaliser sur la réforme de la police dans ce pays. En examinant la transition du régime militaire vers un régime démocratique en Guinée, Dominique Bangoura explique en détail comment les acteurs militaires favorables aux réformes se sont accordés avec des politiciens civils pour réorganiser léquilibre du pouvoir de manière formelle et informelle - entre les forces armées et un gouvernement démocratique nouvellement élu.

Les auteurs traitent également d'une deuxième dimension des moments potentiellement transformationnels de la réforme de la gouvernance et du secteur de la sécurité: il s'agit de la nature de la prestation des services de sécurité par l'Etat. Pour qu'il y ait une bonne gouvernance du secteur de la sécurité, l'Etat doit répondre aux besoins de sécurité ressentis par la population en mettant l'accent sur les mandats, la redevabilité, l'efficacité et l'efficience des organisations chargées du recours à la force au nom de l'Etat; l'Etat doit aussi rendre compte de la manière dont il assume sa responsabilité de surveillance et de contrôle de ce recours à la force. Ces aspects de la réforme sont les plus susceptibles de refléter des changements (pas nécessairement positifs) à court terme, et ce du simple fait que les institutions de sécurité sont souvent un point d'entrée pour les réformes. Dans cette série détudes de cas, E. Remi Aiyede et Zeïni Moulaye montrent comment, respectivement au Nigeria et au Mali, les dysfonctionnements des rapports entre gestion, contrôle et performance des services de sécurité ont contribué aux lacunes opérationnelles des forces de défense. Pour sa part, le général Lamine Cissé offre un point de vue personnel sur la manière dont les forces de défense au Sénégal ont, à ses yeux, pu éviter dêtre politisées alors même qu'elles étaient confrontées à la double pression d'une insurrection interne et de la consolidation délicate de la démocratie multipartite. En sécartant d’autres analyses de ce conflit au Sénégal, en particulier en ce qui concerne le respect des droits humains et le traitement de la population civile, ce chapitre illustre également le fossé qui sépare les perspectives internes et externes sur une situation donnée.

Une autre dimension importante de ces études de cas est qu'elles ne s'attachent pas uniquement aux succès. Plusieurs contributions montrent comment des opportunités de réforme et d'amélioration de la gouvernance ont pu être bloquées, contrecarrées ou sabotées par des intérêts particuliers ou du fait dévénements contingents: Aiyede montre bien comment la corruption et les intérêts particuliers ont étouffé lélan du processus de réforme de la défense au Nigeria. Zeïni Moulaye montre quant à lui comment, au Mali, la stratégie de réforme exhaustive et globale est restée lettre morte du fait de manœuvres politiques, ce qui a finalement contribué à créer les conditions de la crise nationale qui a éclaté en 2012.

La conclusion de ce volume présente les leçons à tirer en matière de RSS à partir d'une analyse comparative de ces riches études de cas. Elle souligne la pertinence d'appréhender la réforme comme un processus itératif et progressif de transformation de la dynamique de la gouvernance du secteur de la sécurité. 
Cette perspective peut révéler, aux niveaux national, sous-national et local, des aspects - aussi bien positifs que négatifs - qui pourraient sinon rester invisibles. Une analyse détaillée de la dynamique de la réforme peut alors permettre d'élaborer des stratégies mieux adaptées au contexte local tout en identifiant des priorités de réforme plus constructives. Cette approche analytique permet également de tirer d'autres leçons des vastes expériences en matière de RSS engrangées en Afrique de l'Ouest et de réfléchir avec une nouvelle optique à la manière de soutenir les réformes qui visent à améliorer la gouvernance du secteur de sécurité.

\section{Tirer des leçons des expériences ouest-africaines en matière de gouvernance du secteur de la sécurité}

En réunissant six éminents experts pour rendre compte de divers moments dans la longue trajectoire des processus de réforme dans leur pays respectifs, ce volume offre une vision unique des expériences de réforme dans chacun de ces contextes nationaux. Chacun de ces auteurs s'est focalisé sur des moments spécifiques de mutations politiques qui ont conduit soit à un changement qualitatif dans certains aspects de la gestion, de la surveillance et du contrôle du secteur de la sécurité, soit à un changement dans la nature de la prestation des services de sécurité. Cette approche met particulièrement l'accent sur la manière dont a émergé, dans chacune de ces situations, l'élan qui est venu soutenir ou entraver le processus de réforme.

Le chapitre 2 examine la réémergence du Conseil de police au Ghana en 1992. Le Ghana est souvent cité, dans la région, comme un exemple en matière de consolidation démocratique et de renforcement de la gouvernance du secteur de sécurité, mais les ressorts de cette évolution restent mal compris. Comblant cette lacune dans la connaissance historique de la gouvernance démocratique au Ghana, Aning examine la réémergence du Conseil de la police, en 1992, ainsi que son bilan inégal en tant qu’organe de contrôle démocratique et de gestion de la police avant et depuis ce tournant. En retraçant la genèse de cet organe durant la phase démocratique ayant suivi l'indépendance du Ghana, puis sa marginalisation durant la dictature militaire jusqu'à sa résurrection avec la Constitution de 1992, le chapitre 2 souligne la pertinence d'examiner les avancées en matière de transformation démocratique par le point d'entrée des micro-dynamiques de processus de réforme de plus grande ampleur sur le plus long terme.

Le chapitre 3 examine la transition politique en Guinée. Dans ce pays, l'héritage lourd et tourmenté qu'a laissé le régime militaire donnait peu d'espoir pour l'avenir, lorsqu'un soldat jeune et inexpérimenté a brusquement pris le pouvoir en 2008. Pourtant, ce contexte sombre a, peu à peu, débouché sur une transition démocratique pilotée par un responsable militaire convaincu de la 
nécessité de procéder à des réformes. Celui-ci a réussi à réduire le fossé entre civils et militaires en instaurant un climat de confiance et en posant les bases d'une nouvelle ère politique. Mettant l'accent sur la phase délicate de transition politique qu’a connu la Guinée au cours de la période 2009-2010, Dominique Bangoura retrace le difficile retour à un gouvernement civil et l'instauration d'un contrôle civil et démocratique sur le secteur de la sécurité.

Le chapitre 4 examine la question négligée de la gouvernance législative de la réforme menée au Libéria après la fin du conflit dans ce pays. Bien que le Libéria ait été dirigé, sans interruption, par un gouvernement civil durant 133 ans, ce pays, qui est l'une des plus anciennes républiques indépendantes de l'Afrique, a néanmoins connu, tout au long de son histoire, de graves déficits démocratiques en matière de gouvernance législative. Après avoir dressé le bilan de deux décennies de régime militaire et d'un conflit dévastateur, Sayndee retrace l'émergence d'un pouvoir législatif libérien et le rôle sans précédent que celui-ci a joué dans la mise en place d'un tout nouveau cadre législatif pour la gouvernance démocratique du secteur de la sécurité dans le contexte du long processus de rétablissement de la paix.

Le chapitre 5 explore le bilan de la réforme au Mali durant sa phase de transition démocratique. Considéré comme un modèle de réforme transformationnelle et de prévention pacifique des conflits dans les années 1990, le Mali a connu soudain, en 2012, un revirement du processus démocratique et a vu émerger des conflits armés qui ont surpris de nombreux observateurs. La focale a surtout été portée sur l'état du secteur de la sécurité au Mali depuis les événements dramatiques de 2012, mais Moulaye opère un retour en arrière pour examiner les processus de réforme exhaustifs menés dans les années 2000, et trouve les racines de la crise ultérieure dans les échecs des efforts visant à remettre en cause le confinement du secteur de la défense à un domaine réservé.

Le chapitre 6 dresse le bilan des promesses suscitées par la transition du Nigéria vers la démocratie, qui semblait augurer une nouvelle ère de gouvernance démocratique du secteur de la sécurité. Pourtant, les forces de sécurité du pays ne se sont pas affranchies des dysfonctionnements et des pratiques de corruption qui ont entraîné des lacunes dramatiques en matière de sécurité et de défense nationale, alors même que le pays se trouve confronté à une insurrection interne aux pratiques particulièrement brutales. Aiyede explique les échecs des processus de RSS durant la période 1999-2007 à la lumière des défis sécuritaires auxquels est confronté le Nigeria, en montrant comment le potentiel de réforme s'est transformé en échec.

Le chapitre 7 présente l'expérience du Sénégal en matière de relations civilo-militaires, qui est unique dans la région. Dans ce pays, la longue tradition de démocratie consolidée et pacifique contraste avec les expériences de nombreux autres Etats de la région. Cela est d’autant plus remarquable que le Sénégal a réussi à consolider sa démocratie tout en préservant son secteur de la sécurité des effets déstabilisateurs des conflits armés internes qui ont affaibli nombre 
de ses voisins. Cissé présente son point de vue d'initié pour relater la genèse d'un contrôle civil démocratique au Sénégal au moment où la plus ancienne démocratie de l'Afrique de l'Ouest vivait sa première alternance démocratique dans une nouvelle ère de pluralisme politique et alors même que l'un des plus anciens conflits internes du monde se poursuit en Casamance.

Malgré ce large éventail de contextes nationaux, un certain nombre de points communs émergent cependant. Des décennies de gouvernance autoritaire ont laissé en héritage des cultures spécifiques de la sécurité qui présentent des caractéristiques communes dans les six contextes étudiés. Ainsi, dans chacun des pays étudiés, la sécurité est traitée comme un domaine tabou réservé aux professionnels de la sécurité, ou un peu plus largement, à lélite politique. Cela a également contribué à une culture de rapports conflictuels entre les forces de sécurité et l'exécutif politique, entre l'exécutif politique et d’autres branches du gouvernement et entre le gouvernement et la population telle que représentée par la société civile. En outre, les questions de sécurité sont très rarement discutées au-delà de petits cercles d'initiés. Cela reflète la priorité accordée à la sécurité du régime et la tradition de considérer les institutions de sécurité comme les défenseurs du pouvoir étatique. Ces caractéristiques ont eu plusieurs conséquences directes pour la RSS, qui sont mises en évidence dans l’analyse comparative figurant dans le chapitre 8 qui conclut ce volume.

Après une décennie et demie de soutien externe ciblé et de prescriptions parfois imposantes, le bilan des processus de RSS est, à ce jour, au mieux contrasté et il est clair que ces processus ne se sont pas déroulés comme escompté. Les défis que la RSS cherche à surmonter peuvent se résumer à léchec des tentatives de lutter contre les modèles dysfonctionnels de gouvernance de la sécurité qui maintiennent le statu quo dans les relations de pouvoir et sapent la légitimité de l'Etat en tant que prestataire de services de sécurité. Si la complexité inhérente à la gouvernance du secteur de la sécurité dans un contexte national donné peut expliquer, en grande partie, pourquoi la RSS n’a pas entraîné des changements transformationnels, la conclusion de ce volume soutient que cet échec découle partiellement de l'absence d'une perspective analytique à même d'identifier les transformations itératives et graduelles de la gouvernance. Les études de cas qui suivent visent à remédier à cette lacune en offrant des analyses détaillées des changements lents et fragiles de la gouvernance du secteur de sécurité qu’a connus l'Afrique de l'Ouest.

\section{Notes}

${ }^{1}$ En se focalisant sur les moments de changement et sur l'influence de différents acteurs dans les processus séquentiels de lévolution institutionnelle, l'approche analytique adoptée dans cet ouvrage se fonde sur les théories historiques et sociologiques de l'institutionnalisme. Pour les références clés 
sur cette approche, voir Hall et Taylor (1996); March et Olsen (1983); Peters (2011).

2 Alors que l'Indonésie et l'Afrique du Sud constituent toutes deux des exemples de leadership national fort, il convient de noter que, dans les deux cas, les acquis de la réforme dans l'ère post-transition ont été, par la suite, remis en cause et considérés comme n'ayant pas pu s'adapter à la notion normative essentielle de la RSS relative aux principes de la bonne gouvernance dans le secteur de la sécurité. Pour des interprétations alternatives sur cette question, consulter Baker (2015); Altbeker (2005). 